\title{
均分散异形微粉 $\alpha-\mathrm{Fe}_{2} \mathrm{O}_{3}$ 的研究
}

魏 雨 郑学忠 刘晓林 郡素霞 鹿 平

(河北师范大学化学系、实验中心, 石家庄 050016)

\section{关键词 $\alpha-\mathrm{Fe}_{2} \mathrm{O}_{3}$ 异形微粉 水热反应法}

均分散异形微粉在现代高技术材料领域有着广泛的应用前景. 尤其是纺锤形和针形 $\alpha-\mathrm{Fe}_{2} \mathrm{O}_{3}$ 的出现, 标志着磁记录材料的重大发展 ${ }^{[1,2]}$. 由于它特殊的形状及颗粒的微细化, 是实 现高密度记录的理想材料, 近年来倍受人们的重视.

水热法制备异形 $\alpha-\mathrm{Fe}_{2} \mathrm{O}_{3}$ 微粉是一条重要途径 ${ }^{[3] 1)}$, 但由于诸多的影响因素, 人为的控制颗 粒大小及形状和实验结果的重现性极差, 是实现工业化生产的主要障碍. 我们从控制颗粒的 形状及重现性人手, 采用水热法引人不同的晶体助长剂, 反应液采用搅拌和静止陈化的环 境. 实现了实验结果的重现性并制备出了符合要求的特殊形状 (如针形、纺锤形、椭球形和短 柱形)的微粉, 在探索异形微粉工业化生产中有所突破.

\section{- 1 实验和结果}

\section{1 试剂及装置}

实验中使用的 $\mathrm{Fe}\left(\mathrm{NO}_{3}\right)_{3} \cdot 9 \mathrm{H}_{2} \mathrm{O}, \mathrm{NaOH}, \mathrm{NaH}_{2} \mathrm{PO}_{4}$ 的柠檬酸均为 A.R. 级试剂, 1- 差基亚 乙基 - $1,1^{\prime}$-二膦酸 (HEDP) 按文献[4] 制备. 实验时采用 $2 \mathrm{~L}$ 不锈钢高压反应金.

\section{2 实验方法}

用普通的去离子水配制一定浓度的 $\mathrm{Fe}\left(\mathrm{NO}_{3}\right)_{3}$ 溶液, 加人 $10 \%$ 的 $\mathrm{NaOH}$ 使其 $\mathrm{pH}$ 值为 7.5 7.8. 将 $\mathrm{Fe}(\mathrm{OH})_{3}$ 沉淀升温至 $60 \sim 65^{\circ} \mathrm{C}$ 后保温约 $3 \mathrm{~min}$, 过滤、水洗滤饼. 然后用水重新 分散滤饼, 分散后的体积约是 $\mathrm{Fe}\left(\mathrm{NO}_{3}\right)_{3}$ 溶液的 $1 / 2$. 加人晶体助长 剂, 最后用 $5 \%$ 的 $\mathrm{NaOH}$ 溶液调节 $\mathrm{pH}$ 为 $10.0 \sim 10.5$ 后人金. 约 $90 \mathrm{~min}$ 升温至反应温度, 反应结束后沉淀过 滤、水洗并烘干. 取样分散于水中, TEM 观测粒子的形貌. 用 CuK $\alpha$ 对 所得试样进行物相分析, XRD 图谱 如图 1 所示, 证明为 $\alpha-\mathrm{Fe}_{2} \mathrm{O}_{3}$. 实验条 件及相应的 TEM 照片见表 1 和图 2.

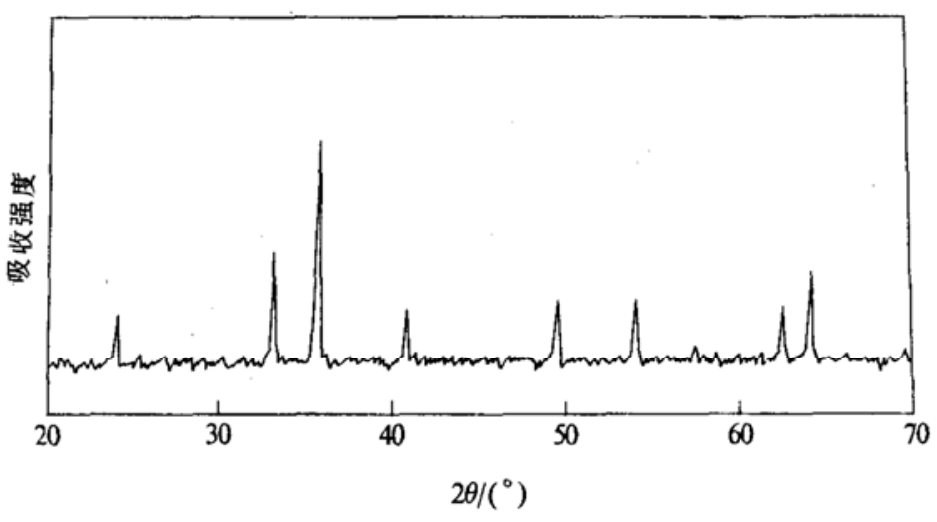

图 1 表 1 中试样 (d) 的 XRD图

1995-05-03 收稿, 1995-09-21 收修改稿

1) 魏雨, 郑学忠. 均分散针状 $\alpha-\mathrm{Fe}_{2} \mathrm{O}_{3}$ 的制备. 应用化学, 1996 (待发表) 
表 $1 \alpha-\mathrm{Fe}_{2} \mathrm{O}_{3}$ 的制备条件及结果

\begin{tabular}{|c|c|c|c|c|c|c|c|}
\hline 编 & \multicolumn{2}{|c|}{ 反应物组成 $/ \mathrm{mol} \cdot \mathrm{L}^{-1}$} & \multirow{2}{*}{ 反应温度/ ${ }^{\mathrm{C}}$} & \multirow{2}{*}{ 反应环境 } & \multirow{2}{*}{ 反应时间/h } & \multirow{2}{*}{ 粒子形状 } & \multirow{2}{*}{ 轴 比 } \\
\hline 号 & $\mathrm{Fe}(\mathrm{OH})_{3}$ & 晶体助长剂 & & & & & \\
\hline (a) & 0.30 & 柠檬酸 $1 \times 10^{-3}$ & 170 & 静态 & 3 & 短柱状 & $1.5 \pm 0.3$ \\
\hline (b) & 0.32 & HEDP $1 \times 10^{-3}$ & 170 & 静态 & 3 & 椭球状 & $1.4 \pm 0.2$ \\
\hline (c) & 0.30 & $\mathrm{NaH}_{2} \mathrm{PO}_{4} 1.2 \times 10^{-3}$ & 170 & 摚拌 $\left(500 \mathrm{r} \cdot \min ^{-1}\right)$ & 2 & 纺锤状 & $3.5 \pm 0.5$ \\
\hline (d) & 0.30 & HEDP $1 \times 10^{-3}$ & 180 & 摚拌 $\left(500 \mathrm{r} \cdot \mathrm{min}^{-1}\right)$ & 2 & 针 状 & $7 \pm 0.5$ \\
\hline
\end{tabular}

由表 1 和图 2 可以看出, 加人不同的晶体助长剂及实验条件不同时, 制取的 $\alpha-\mathrm{Fe}_{2} \mathrm{O}_{3}$ 微粒 有很大的差异. 加人有机磷酸 (HEDP) 在摚拌环境下可明显增加微粒的轴比, 该微粒可制取 理想轴比的 $\gamma-\mathrm{Fe}_{2} \mathrm{O}_{3}$ 磁粉. 加人 $\mathrm{NaH}_{2} \mathrm{PO}_{4}$ 作为晶体助长剂时, $\alpha-\mathrm{Fe}_{2} \mathrm{O}_{3}$ 为纺锤形, 是无极微粉 的原料, 与强迫水解法 ${ }^{[2]}$ 极为相似.
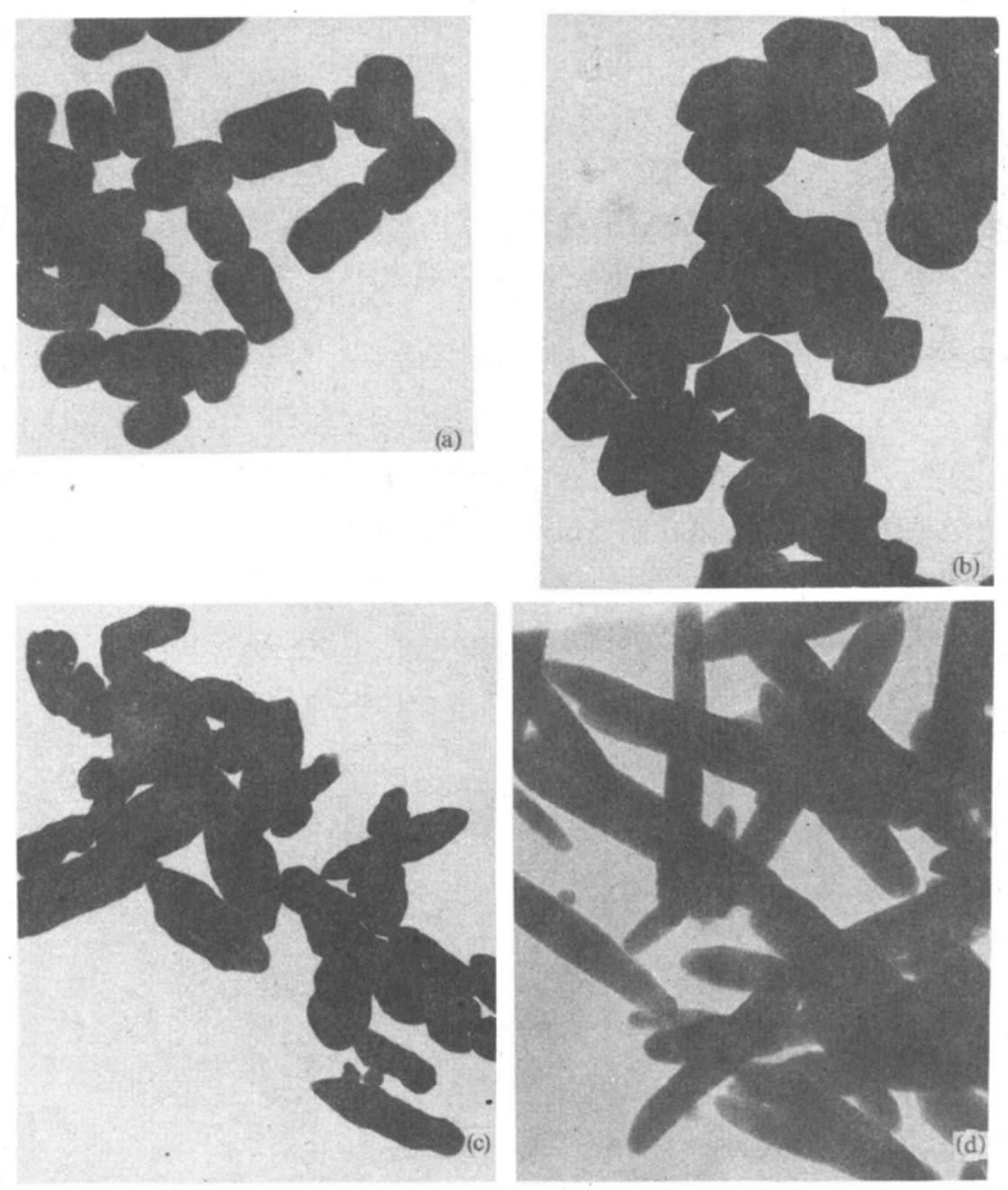

图 $2 \alpha-\mathrm{Fe}_{2} \mathrm{O}_{3}$ 微粒的 TEM 照片

(a) $\times 60 \mathrm{~K}$, (b) $\times 80 \mathrm{~K}$, (c) $\times 30 \mathrm{~K}$, (d) $\times 80 \mathrm{~K}$. 对应于表 1 中的实验编号 


\section{2 讨论}

(1) 由 $\mathrm{Fe}(\mathrm{OH})_{3}$ 凝胶在碱性条件下水热制备异形微粉 $\alpha-\mathrm{Fe}_{2} \mathrm{O}_{3}$, 在不同的反应时间取样, TEM 观测、XRD 物相分析证明其形成机理为

$$
\mathrm{Fe}(\mathrm{OH})_{3} \stackrel{(\mathrm{l})}{\longrightarrow} \alpha-\mathrm{FeOOH} \stackrel{(2)}{\longrightarrow} \alpha-\mathrm{Fe}_{2} \mathrm{O}_{3}
$$

该反应的反应速率不但取决于 $\mathrm{Fe}(\mathrm{OH})_{3}$ 凝胶的初始浓度、 $\mathrm{pH}$ 值, 而且与加人晶体助长剂的种 类、浓度以及反应温度有关. 表 1 中 (b) 和 (d) 加人相同的晶体助长剂 HEDP 和浓度 $1 \times 10^{-3} \mathrm{~mol} \cdot \mathrm{L}^{-1}$, 但当反应温度由 $170^{\circ} \mathrm{C}$ 提高到 $180^{\circ} \mathrm{C}$ 时, 完全转化为 $\alpha-\mathrm{Fe}_{2} \mathrm{O}_{3}$ 的反应时间由 $3 \mathrm{~h}$ 减为 $2 \mathrm{~h}$, 这说明提高反应温度可明显缩短 $\alpha-\mathrm{FeOOH}$ 相转化为 $\alpha-\mathrm{Fe}_{2} \mathrm{O}_{3}$ 的时间.

(2) 实验证明一定的摚拌速度有利于生成均匀针形的 $\alpha-\mathrm{Fe}_{2} \mathrm{O}_{3}$ 粒子. 这由表 1 中 (b) 和 (d) 可以得到证实. 晶体助长剂的加人与早期生成的晶核 $\alpha-\mathrm{Fe}_{2} \mathrm{O}_{3}$ 络合, 摚拌使得反应后期由 $\alpha-\mathrm{FeOOH}$ 相转化为 $\alpha-\mathrm{Fe}_{2} \mathrm{O}_{3}$ 靠络合物空间构型的影响, 沿着一定的方向生长成所需的晶形 1 .

\section{3 结论}

研究证明, 水热法制备均分散异形微粉 $\alpha-\mathrm{Fe}_{2} \mathrm{O}_{3}$, 加人不同的晶体助长剂、控制不同的反应 条件, 可生成形状各异的 $\alpha-\mathrm{Fe}_{2} \mathrm{O}_{3}$ 微粉. 晶体助长剂 HEDP 有利于 $\alpha-\mathrm{Fe}_{2} \mathrm{O}_{3}$ 微粒长成针形. 实验结果实现了特殊形状和晶形的异形微粉的重现性. 实验中没有采用二次蒸馏水, 没有使 用微孔滤膜过滤及特殊的防尘设备, 向异形微粉工业化生产迈进了一步.

\section{参考文献}

1 Ishikawa T, Matijević E. Formation of monodispersed pure and coated spindle-type iron particles. Langmuir, 1988, 4: 26

2 任福民, 曾桓兴. 强迫水解法制备纺锤形 $\alpha-\mathrm{Fe}_{2} \mathrm{O}_{3}$ 微粒的研究. 科学通报, 1991, 36(8):627

Subrt J, Stengl V. Preparation of acicular $\alpha-\mathrm{Fe}_{2} \mathrm{O}_{3}$ (haematite). J Mater Sci Lett, 1993, 12:836

4 朱传方,张景龄,卢金桥. 新金属配位体 1- 羟基亚乙基二膦酸的合成与纯化. 化学试剂, 1991，13(2):125

5 Arndt V. $\gamma-\mathrm{Fe}_{2} \mathrm{O}_{3}$ of improved quality from direct synthesis of acicular $\alpha-\mathrm{Fe}_{2} \mathrm{O}_{3}$. IEEE Trans Magn, MAG-24, 1988, 1796 